\title{
Clinical management of childhood sleeps bruxism
}

\section{Background}

Introduction: Sleep bruxism (SB) affects between $3.5 \%$ and $46 \%$ of children from 0 to 12 years of age and can cause damage to the stomatognathic system and the various structures that are part of it.

Objective: this paper aims to report a clinical case of childhood SB regarding the origin, signs, symptoms, the conduction and clinical management of it, minimizing the possible consequences on the child's oral health-related quality of life.

Description: a 6-year-old female patient (L.T.B.) presented to the Pediatric Dentistry Clinic of the Aeronautic Hospital of Canoas (HACO) with a complaint of headache and clenching. The diagnosis of SB was obtained through parental report, and it was believed to be caused by respiratory problems triggered by weather changes. Therefore, no muscle or joint anomalies were identified. Multidisciplinary treatment was carried out with the otorhinolaryngologist, as well as the application of a rigid acrylic occlusal spint by the pediatric dentist.

Conclusion: In this case report, an occlusal splint was a favorable therapy for the resolution of dental wear caused by SB in the deciduous dentition. It was also reported by the parents and improvement on the child's sleep routine, providing a better oral health-related quality of life to the patient.

Keywords: sleep bruxism, children, pediatric dentistry
Volume II Issue 2 - 2020

\author{
Claudia Britto Azevedo, ${ }^{2}$ Fernando Valentim \\ Bitencourt,' Natália Sabadin,' 'Vitória de \\ Oliveira Chami, ${ }^{3}$ Michelle Roxo-Gonçalves, ${ }^{4}$ \\ Paola Arosi Bottezini ${ }^{5}$ \\ 'Department of Conservative Dentistry, Federal University of \\ Rio Grande do Sul (UFRGS), Brazil \\ ${ }^{2}$ Department of Pediatric Dentistry, Federal University of Rio \\ Grande do Sul (UFRGS), Brazil \\ ${ }^{3}$ Doctoral Program in Dental Sciences/Orthodontics, Federal \\ University of Santa Maria, Brazil \\ ${ }^{4}$ Department of Oral Pathology, Federal University of Santa \\ Maria, Brazil \\ ${ }^{5}$ Faculty of Dentistry, Federal University of Rio Grande do Sul, \\ Porto Alegre, Brazil
}

Correspondence: Fernando Valentim Bitencourt Departmen of Conservative Dentistry, Federal University of Rio Grande do Sul (UFRGS), Brazil, Tel 5551999037724,

Email Fernando.bitencourtxd@gmail.com

Received: March 06, 2020 | Published: March 13, 2020
Abbreviations: SB, sleep bruxism; HACO, aeronautic hospital of canoas; CNS, central nervous system

\section{Introduction}

Even at a young age, children can develop harmful oral habits that may lead to an imbalance between growth and function. Sleep bruxism (SB), in particular, affects between $3.5 \%$ and $46 \%$ of children from 0 to 12 years of age ${ }^{1}$ and can cause multiple damages to the stomatognathic system and to the various physical structures that are part of it.

Although it's considerable prevalence, diagnosis and treatment of SB still raises doubts for clinicians and researchers. SB is defined as a parafunctional activity of the masticatory muscles, which differs through the circadian moment in which the event takes place: during sleep (SB) or in waking state (awake bruxism). ${ }^{2}$ Such parafunction is not seen as a disorder, but rather as a motor behavior of multifactorial etiology, which turns out to be a sign of some other health condition. ${ }^{3,4}$

The signs and symptoms of bruxism can have a functional impact related to dental and craniofacial changes, as well as emotional and psychological impact, such as stress and anxiety, on children's lives, directly affecting quality of life. ${ }^{5,6}$ Neverthless, until the present moment, no therapy has been shown to be effective in controlling SB, mainly due to the multiplicity of the phenomena associated with this parafunctional habit, a fact that hinders the therapeutic approach and the detection of its etiological factors. ${ }^{7}$

Thus, knowing that undiagnosed or untreated oral and dental changes can cause disturbances in physical, psychological and social performance, compromising simple activities on a child's daily routine, the aim of this report is to introduce a clinical case in which dental surgeons can acquire enough information in order to identify, in the clinical context, the origin, signs and symptoms and perform clinical management of childhood SB, objecting to minimize its possible consequences on the child's oral-related quality of life.

\section{Clinical report}

This clinical report followed the CARE guidelines for improving the quality of reports of case report. ${ }^{8}$ The study was conducted in accordance with the Helsinki Declaration of 1975 and the authorization term for execution of the proposed treatment and use of image was accepted and signed by the parents. The guardians of L.T.B., a 6 years old female patient, sought care at the Section of Pediatric Dentistry of the Dental Division of the Aeronautic Hospital of Canoas (HACO), in May of 2019, with the complaint that their daughter was making a loud noise, similar to teeth grinding, every night, for 6 months in the course of sleep. It was also reported that the child presented headaches daily.

During the anamnesis, when asked about the child's general health status, the mother reported that the girl was in good general health and did not use medication for continuous use. However, during the winter and spring months, mainly, the patient presents frequent allergic episodes, but had never been taken to be examined by an otolaryngologist.

In addition, the parents described that their daughter had current headaches with no apparent reasons. A very agitated behavior and restless sleep were reported. Psychological help was sought in order to deal with the daughter's attitudes at home, but after professional evaluation no behavioral disorder was found. 
During clinical examination of the extraoral structures, the child did not present changes to the palpation performed on the orofacial muscles, especially those associated with the act of chewing, and on the TMJ. However, during intraoral examination, the patient, who had complete deciduous denture, presented intense tooth wear on the occlusal surfaces of molars and incisal edges of upper and lower deciduous canines, in proximity to the dentin (Figures $1 \& 2$ ).

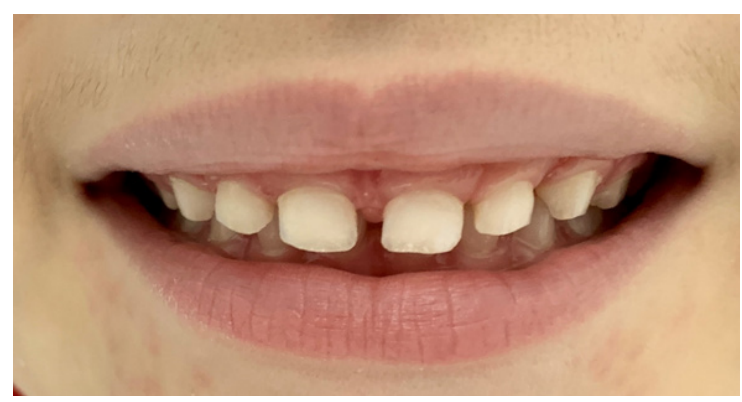

Figure I Frontal smile.

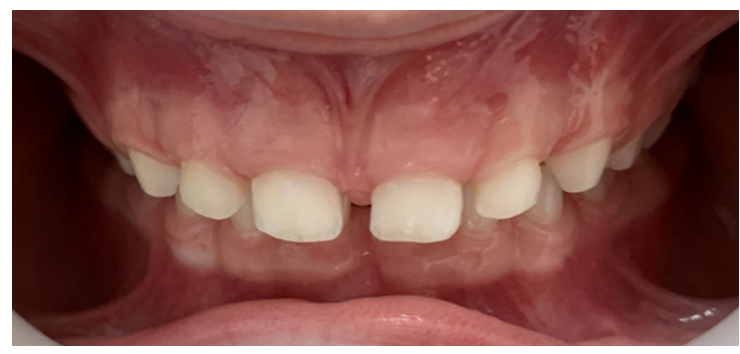

Figure 2 Presence of wear on the incisal edges of elements 53 and 63.

Thus, SB was diagnosed through parental report and the parafunction's signals found in the patient's physical structures. The guardians were instructed to seek an evaluation of the otorhinolaryngological structures with the specialist in the corresponding medical field. At the return visit, the mother reported that the child was diagnosed with seasonal allergic rhinitis, and that the medical treatment would begin soon.

The clinical conduction of the case was based on the prevention of a more severe wear of the remaining dental structures, therefore, an acrylic occlusal rigid splint was planned to be used at home, during sleep. In order to increase the durability of the device, an expander screw was added, since the patient is experiencing physical growth (Figure 3).

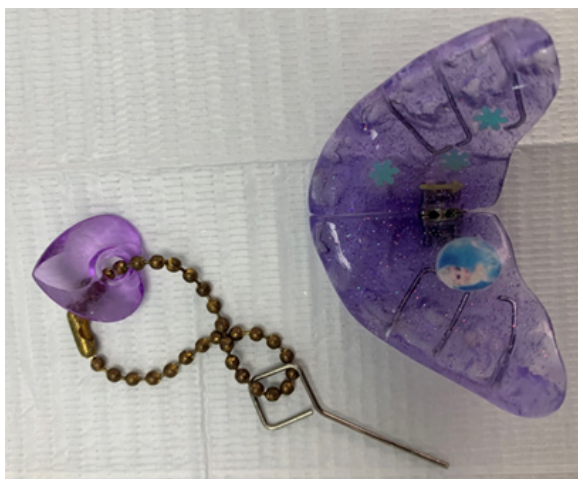

Figure 3 Smooth and rigid acrylic occlusal plate. Presence of expansion screw and activation key.
For that reason, the patient had its dental arches molded with Hydrogum 5 alginate from Zermack (Badia Polesini, Italy) and the bite record marked in wax 7 Lysanda ${ }^{\circledR}$ (São Paulo, Brazil). The material was sent to the prosthetic laboratory of choice of the guardian. Maintenance appointments and adjustments of the occlusal plaque were carried out monthly, in order to maintain the coverage of the occlusal and incisal surfaces of the teeth of the upper arch, with the presence of bilateral, simultaneous and stable contacts with the opposite arch. Also, the expansion screw was activated by the dentist in each visit in order to monitor the craniofacial growth and the perimeter of the arches.

After 2 months of use, it was possible to observe the presence of significant wear on the occlusal splint, which attests to its correct indication, as well as the child's good adherence to the treatment. The parents reported that the child showed an improvement in sleep, fewer reports of headache, and a little change in behavior and oral-health related quality of life.

\section{Discussion}

Bruxism is a para functional activity of the orofacial musculature, especially of the masticatory muscles, which consists of the habit of clenching or grinding the teeth during sleep or during awakeness. ${ }^{2}$ Even with this difference, SB and awaken bruxism are considered two distinct entities, with their genesis in the central nervous system $(\mathrm{CNS})^{7}$ and are modulated by several neurochemical substances, such as adrenergic, noradrenergic and serotonergic neurotransmitters. ${ }^{9}$

In $\mathrm{SB}$, rhythmic masticatory muscle activity is associated with an episode of adrenergic micro-awakening and increased heart rate during the REM sleep phase. ${ }^{7}$ In people who are considered healthy, this parafunction is not seen as a disorder, but as a motor behavior of multifactorial etiology, which is a sign of some other health conditions, as observed in the case described above. ${ }^{3,4}$

The diagnosis of SB in children is still a challenge, since the main tool for assessing this condition in childhood is the report of parents or guardians. ${ }^{8}$ In addition, a physical examination of dental and facial muscle structures is recommended. ${ }^{2}$ Polysomnography, the gold standard for SB diagnosis, ends up being less used in this age group due to the difficulty of its application, which is performed in a hospital environment, generating a discomfort for the infant patient, and also a high financial cost. ${ }^{10,11}$

However, it is considered sufficiently acceptable that the diagnosis of SB in children is carried out through the report of the parents or guardians, since a previous study identified that the narrative of teeth clenching/grinding was coincident with $83 \%$ of the confirmed cases with the polysomnography when the parents were more informed about the signs and symptoms of SB. ${ }^{12,13}$ In this manner, the presence of the habit cannot be definitively attested, but the parents' report and a thorough examination of the patient and anamnesis lead dental clinicians to a probable scenario of its occurence.

The most frequent symptoms of SB in children are headaches and muscles tiredness, drowsiness, difficulty of concentrating, hyperactivity, anxiety, aggression and attention deficit, ${ }^{14,15}$ in addition to negative oral experiences, such as exacerbated tooth wear, fractures of restorations, pain and discomfort in the temporomandibular joint and hypertrophy of the masseter muscle, factors that can lead to a negative impact on oral-related quality of life. ${ }^{16}$

Occlusal splints are widely used in clinical practice, but there is 
insufficient evidence to state that it is effective for controlling SB However, it acts by protecting teeth against dental wear, removing occlusal interference and relaxing masticatory muscles. ${ }^{17}$ The application of behavioral strategies, such as sleep hygiene, relaxation techniques and removal of risk factors (caffeine and stimulating teas, for example) have been used as the first step in the therapeutic intervention of SB, but with low clinical evidence of its effectiveness. ${ }^{13}$

In this case report, it can be observed that even if the use of occlusal splint does not interrupt the habit, there is still the conservation of dental tissues, avoiding further damage to the structures of the stomatognathic system, which is still in phase of development.

\section{Conclusion}

When faced with a case of childhood SB, it is essential to identify its origin, analyze its signs and symptoms and perform the appropriate clinical management of it, adapting it to the patient's age. In this case report, the occlusal splint was presented as an excellent therapy for the resolution of dental wear caused by such para functional habit. Sleep quality and the end of recurrent headaches episodes were also positive aspects reported by parents after treatment.

\section{Funding}

None.

\section{Acknowledgments}

None.

\section{Conflicts of interest}

The authors declare that there are no conflicts of interest.

\section{References}

1. Machado E, Dal-Fabbro C, Cunali PA, et al. Prevalence of sleep bruxism in children: a systematic review. Dental Press Journal of Orthodontics. 2014;19(6):54-61.

2. Lobbezoo F, Ahlberg J, Raphael KG, et al. International consensus on the assessment of bruxism: Report of a work in progress. J Oral Rehabil. 2018;45(11):837-844

3. Manfredini D, Guarda-Nardini L, Marchese-Ragona R, et al. Theories on possible temporal relationships between sleep bruxism and obstructive sleep apnea events. An expert opinion. Sleep and Breath. 2015;19(4):1459-1465
4. Castroflorio T, Bargellini A, Rossini G, et al. Sleep bruxism and related risk factors in adults: A systematic literature review. Arch Oral Biol. 2017;83:25-32.

5. Ferreira-Bacci, Adriana do Vale, Cardoso, et al. Behavioral problems and emotional stress in children with bruxism. Braz Dent J. 2012;23(3):246-251.

6. Antunes LA, Castilho T, Marinho M, et al. Childhood bruxism: related factors and impact on oral health-related quality of life. Spec Care Dentist. 2016;36(1):7-12.

7. Koyano K, Tsukiyama Y, Ichiki R, et al. Assessment of bruxism in the clinic. J Oral Rehabil. 2008;35(7):495-508.

8. Gagnier JJ, Kienle G, Altman DG, et al. The CARE Guidelines: Consensus-based Clinical Case Reporting Guideline Development. Glob Adv Health Med. 2013;2(5):38-43.

9. Lobbezoo F, Naeiie M. Bruxism is mainly regulated centrally, not peripherally. J Oral Rehabil. 2001;28(12):1085-1091.

10. Lavigne GJ, Kato T, Kolta A, et al. Neurobiological mechanisms involved in sleep bruxism. Critical Reviews in Biology Medicine. 2003; 14(1):30-46.

11. Restrepo C, Manfredini D, Castrillon E, et al. Diagnostic accuracy of the use of parental-reported sleep bruxism in a polysomnographic study in children. International Journal of Paediatric Dentistry.2017;27(5):318-325.

12. Casset E, Réus JC, Stuginski-Basbosa J, et al. Validity of different tools to asess sleep bruxism: a meta-analysis. J Oral Rehabil. 2017;44(9):722-734.

13. Huynh NT, Desplats E, Bellerive A. Sleep bruxism in children: sleep studies correlate poorly with parental reports. Sleep Medicine. 2016;19:63-68.

14. Carra Yap AU, Chua AP. Sleep bruxism: current knowledge and contemporary management. J Conserv Dent. 2016;19(5):383-389.

15. Mota-Veloso I, Celeste RK, Fonseca CP, et al. Effects of attention deficit hyperactivity disorder signs and socio-economic status on sleep bruxism and tooth wear among schoolchildren: structural equation modelling approach. International Journal of Paediatric Dentistry. 2017;27(6):523-531.

16. Firmani M, Reyes M, Becerra N, et al. Sleep Bruxism in children and adolescents. Rev Chil Pediatr. 2015; 86(5):373-379.

17. Solberg WK, Clark GT, Rugh JD. Nocturnal electromyographic evaluation of bruxism patients undergoing short term splint therapy. $J$ Oral Rehabil. 1975;2(3):215-223. 